\title{
Inverted left atrial appendage following cardiac surgery
}

\begin{abstract}
58-year-old male underwent valve sparing ascending aorta replacement (Yacoub). During surgery, direct postoperative transoesophageal echocardiograpphy revealed a left atrial mass; the left atrial appendage could not be visualised (figure 1 , Post), in contrast to the echocardiogram performed before onset of surgery (figure 1, Pre). The following day, transoesophageal echocardiography was repeated and demonstrated absence of a left atrial mass but again showed the presence of the left atrial appendage (Day 1), suggestive of a temporarily inverted left atrial appendage.

An intracardiac thrombus was considered but was thought to be unlikely because of heparinisation during surgery. Moreover, an inverted left atrial appendage has previously been shown to mimic an intracardiac mass. ${ }^{1}$
\end{abstract}
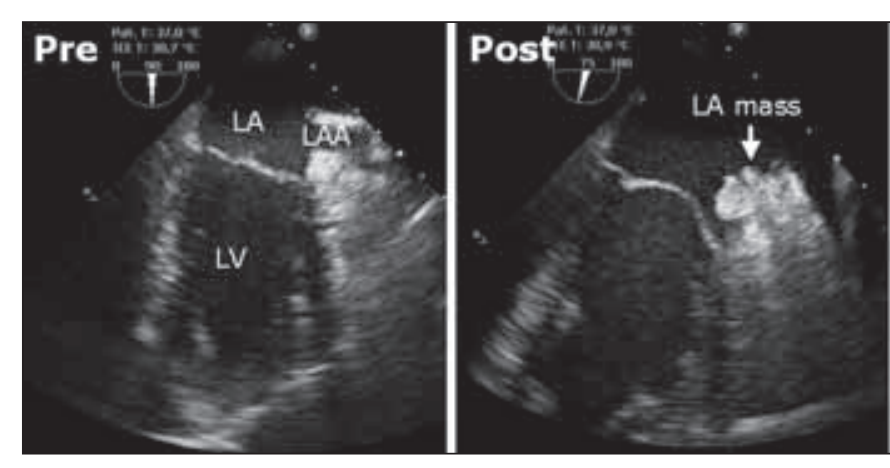

K. Vernooy, J. Vainer, E.C. Cheriex

Department of Cardiology, Maastricht University

Medical Center, Maastricht, the Netherlands J. Nijs

Department of Cardiothoracic Surgery, Maastricht University Medical Center, Maastricht, the Netherlands

Correspondence to: K. Vernooy Department of Cardiology, Maastricht University Medical Center, PO Box 5800,6202 AZ Maastricht, the Netherlands E-mail:kevin.vernooy@mumc.nl

\section{Reference}

1 Chikwe J, Fischer GW, Adams DH. Inverted left atrial appendage. J Am Coll Cardiol. 2009;54:e7.

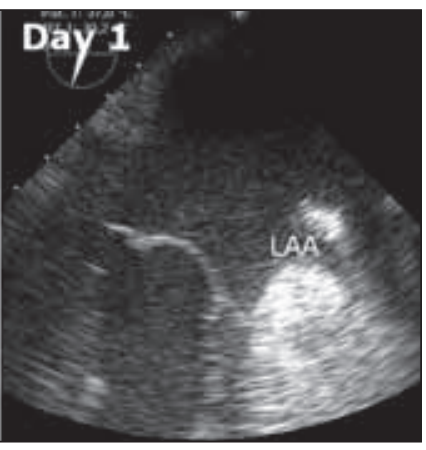

Figure 1. Transoesophageal echocardiographic images revealing an inverted left atrial appendage immediately after cardiac surgery. $L A=$ left atrium, LAA=left atrial appendage, $L V=$ left ventricle. 